FORMATION Formation emploi

Revue française de sciences sociales

97 | janvier-mars 2007

Former pour dynamiser les territoires

\title{
Formation et Territoire : des liens multiples et complexes, mais décisifs
}

André Larceneux

\section{OpenEdition}

1 Journals

Édition électronique

URL : http://journals.openedition.org/formationemploi/1710

DOI : 10.4000/formationemploi. 1710

ISSN : 2107-0946

Éditeur

La Documentation française

Édition imprimée

Date de publication : 1 janvier 2007

Pagination : 81-88

ISSN : 0759-6340

Référence électronique

André Larceneux, "Formation et Territoire : des liens multiples et complexes, mais décisifs ",

Formation emploi [En ligne], 97 | janvier-mars 2007, mis en ligne le 19 février 2009, consulté le 30 octobre 2020. URL : http://journals.openedition.org/formationemploi/1710 ; DOI : https://doi.org/ 10.4000/formationemploi.1710

(c) Tous droits réservés 


\section{Formation et Territoire : des liens multiples et complexes, mais décisifs}

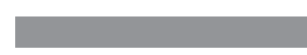

par André Larceneux*

L'inscription territoriale de la formation est devenue une question récurrente sous l'effet d'une double évolution: d'une part, celle des politiques publiques marquées par les mouvements de décentralisation et de déconcentration et, d'autre part, celle des entreprises plus soucieuses, à l'heure de la mondialisation, de leur environnement local.

Les transformations socio-économiques liées à ces évolutions ont donc nécessité un renouvellement des approches scientifiques et des théorisations. Politistes, sociologues et économistes sont ainsi venus sur un terrain d'analyse traditionnellement occupé par les géographes. L'incursion d'un objet nouveau, le «territoire», dans des champs scientifiques disciplinaires cohérents, s'est souvent réalisée dans un flou sémantique et une terminologie ambiguë qui ne favorisent pas la discussion interdisciplinaire pourtant nécessaire. La notion de «territoire», objet complexe, renvoie à une appropriation de l'espace (notion plus physique ou naturelle, mais une nature traduite par des représentations) par des acteurs; elle est dotée d'une polysémie imposée par la pluralité des acteurs et des actions à l'œuvre: politiques sectorielles de l'État, développement des entreprises, mobilités des salariés, comportements des élèves et de leurs familles construisent autant de « territoires » qui se superposent, s'emboîtent et se différencient.
Dans cette optique, le «territoire », construit par les acteurs et leurs représentations, leurs mécanismes cognitifs et leurs institutions, ne saurait avoir une réalité objective suffisante pour imposer une dualité conceptuelle plaçant en interaction équilibrée la formation et le territoire.

Dès lors, qu'est-ce que l'approche par les territoires, notion dont on vient de marquer la complexité, peut apporter à l'analyse de la formation ? Il convient donc dans un premier temps d'expliciter la notion même de territoire, puis la nature des liens entre territoire et entreprises. Enfin, on s'interrogera sur les enjeux de l'approfondissement de ces liens.

* André Larceneux est professeur d'aménagement et d'urbanisme à l'université de Bourgogne, directeur du Laboratoire de géographie humaine de Dijon - ThéMA (Théoriser et modéliser pour aménager)/UMR 6049 CNRS. II a publié récemment avec C. Boiteux-Orain (2006), Paris et ses franges : polycentrisme et étalement urbain, Éditions Universitaires de Dijon. Avec S. Carel (2004), " Microtechniques, Système éducatif et territoire industriel : la difficile reconversion de l'industrie horlogère franc-comtoise » in Formation Emploi, $\mathrm{n}^{\circ}$ 87, La Documentation française, juillet-septembre, pp. 107-1 19 


\section{LE TERRITOIRE COMME RÉFÉRENT THÉORIQUE}

Les géographes distinguent généralement espace et territoire, bien que ces concepts polysémiques puissent être parfois interchangeables. Le premier terme évoque le contenant physique et naturel sur lequel les hommes et les activités se déploient. Un des déterminants de l'espace est la distance entre deux lieux, entre lesquels se déplacent ou interagissent hommes, marchandises, capitaux ou informations. D'une part, si cette distance peut être exprimée de manière objective en unités physiques (mètres, kilomètres,...) elle est soumise aussi à des processus de perception cognitive qui lui affectent des valeurs subjectives: elles définissent le sens de l'éloignement ou de la proximité. Ces processus changent selon les individus, le contexte culturel ou les temporalités. Les économistes ont souvent traduit cette distance en termes de coût généralisé (monétaire ou budget temps) de transport. D'autre part, les relations entre les espaces définis physiquement (reliefs, climats, paysages, distances) et les hommes ou les groupes humains s'y trouvant ont été définies comme des milieux, souvent avec une vision organiciste ou évolutionniste, qui n'a pas toujours été exempte d'une connotation anthropologique douteuse. L'abandon de cette vision anthropologique et l'affaiblissement du rapport conceptuel des groupes humains avec l'espace physique ont orienté la réflexion vers une compréhension plus sociologique, où les jeux d'acteurs sont tributaires non seulement des distances et de leurs perceptions mais aussi des différentes échelles au niveau desquelles se développent ces jeux.

\section{Territoires et échelle : verticalité et horizontalité}

L'action publique doit conjuguer, d'une part, l'égalité (l'équivalence) de droits des citoyens où qu'ils se trouvent sur le territoire national et, d'autre part, une organisation territoriale hiérarchisée : des espaces emboîtés (communes, départements, régions, nation, Europe,...) sont définis comme des aires sur lesquelles s'exercent les compétences de titulaires des pouvoirs de l'action publique. Les individus sont donc confrontés à une double dimension géographique définie par le droit, celle des aires horizontales situées à chaque niveau de compétence administrative. Cette dimension géographique s'exprime par la superposition verticale de niveaux horizontaux qui constituent autant de territoires pertinents pour un acteur. Ils seront confrontés, en termes de politique publique, successivement ou en même temps, aux domaines de compétence des maires, présidents de structures intercommunales, préfets, présidents de conseils généraux, préfets de région, présidents de conseils régionaux, etc.

Ces hiérarchies territoriales sont évidemment multiples et relèvent de l'organisation sectorielle de l'État et des ministères, de la nature de la déconcentration des pouvoirs (région, département, arrondissement) et de l'importance du transfert de compétences aux institutions territoriales décentralisées. Dans le domaine de la formation, les compétences en matière de politique publique sont ainsi réparties entre le ministère, le rectorat et les inspections académiques, la préfecture, les conseils régional et général, les communes. À chacun des niveaux de l'échelle hiérarchique correspond un niveau territorial auquel un acteur de base est confronté. Même pour un seul domaine sectoriel de politique publique (la formation, l'emploi, le logement,...) l'emboîtement de ces territoires est complexe. La notion de « local» (présente dans l'expression « développement local») peut être ainsi définie comme l'interconnexion sur un territoire donné des différents arbres verticaux relevant chacun de politiques sectorielles spécifiques. Du point de vue pratique et d'expérience courante, cela nécessite la réunion, à un niveau géographique donné, d'institutions multiples porteuses soit des différentes composantes sectorielles de la politique de l'État soit des pouvoirs décentralisés : comités de pilotage ou de coordination, réunion ad hoc de concertation, partenariats...

Au sectoriel (ou hiérarchie verticale) s'oppose ainsi le territoire (ou champ horizontal) : ce dernier n'est qu'un lointain produit de l'espace géographique. Il relève plutôt de l'arbitraire historique du découpage administratif et des efforts récents pour faire émerger de nouvelles entités liées à la décentralisation ou à l'intercommunalité. Dans ce cadre, la formation générale comme la formation professionnelle ou les politiques de l'emploi relèvent de l'articulation de 
ces compétences multiples distribuées de manières spécifiques par logiques sectorielles, par types d'institutions (État, collectivités territoriales) et niveaux géographiques. Un bon exemple est celui des «missions locales » qui concentrent et interconnectent de manière souvent difficile tous ces réseaux : les publics doivent être traités en référence à la multiplicité des problèmes rencontrés : emploi, formation, logement, santé, mobilité. Ces publics doivent avoir un lieu, géographiquement déterminé, où ils pourront faire valoir leurs droits et où des solutions leur seront proposées dans une interface réelle avec un référent de l'institution concernée. Cette localisation (qui suppose donc un géoréférencement, une adresse) est la composante géographique du territoire formé de l'ensemble des institutions et de leur champ de compétence.

Le « territoire » est donc ici la traduction de ces interconnections localisées qui s'expriment à travers la distribution des pouvoirs et compétences et dépendent des découpages administratifs.

\section{Des territoires multiples, adaptés à chaque logique d'acteurs}

Géographes et économistes ont essayé de montrer que le fonctionnement des marchés suivait une logique d'emboîtement hiérarchique qui s'apparentait à la hiérarchie administrative. Christaller ( $c f$. Pumain D., Saint Julien Th., 2001, p. 115-120) a pu montrer, dans des analyses anciennes et bien connues, que commerces et services marchands révélaient leurs aires de marchés comme des découpages optimaux (des hexagones) et surtout emboîtés de manière hiérarchique : les médecins généralistes sont disséminés sur l'ensemble du territoire alors que les spécialistes sont fortement concentrés dans les plus grandes agglomérations. Les enquêtes de l'Inventaire communal décrivent ces localisations différentielles des commerces et des «équipements». À l'école communale, présente partout, s'oppose l'université dont est dotée la seule capitale régionale, lycées et collèges ayant une situation intermédiaire. Les aires de recrutement sont, comme des aires de marché, l'expression de la densité des populations et de leurs comportements de mobilité.
La détermination de ces aires est souvent une des approches privilégiées par les géographes: ces derniers mettent l'accent sur les structures hiérarchiques et les réseaux urbains qui s'expriment par ces mobilités des personnes (mais aussi des capitaux ou des informations).

Les mobilités ont aussi fait l'objet d'analyse de la part des économistes, mais ceux-ci ont privilégié une autre démarche. D'une part, ils ont insisté sur le rôle des mobilités domicile/travail. Grâce aux recensements, ils ont pu ainsi délimiter des bassins ou des zones d'emploi à partir des migrations journalières des actifs entre leur domicile et leur lieu de travail. D'autre part, ils ont simplifié le problème pour obtenir un découpage cohérent du territoire. Pour ce faire, ils ont abandonné toute vision hiérarchique des zones d'emploi entre elles, qui auraient pu être intégrées par la nature des migrations (cadres ou employés et ouvriers) ou par les soldes non nuls des migrations interzones. Cet outil des zones d'emploi a pu ainsi fournir une approche originale du « territoire » comme espace d'action de la politique de l'emploi, comme niveau pertinent de la production statistique ou comme domaine de coordination des acteurs. Ce découpage en zones d'emploi a souvent été validé comme territoire économiquement pertinent face à l'arbitraire des découpages administratifs.

En fonction des problèmes traités, d'autres aires de mobilité ont été étudiées : à travers la carte scolaire, la mobilité contrainte des élèves a permis une approche fonctionnelle de la localisation des établissements, à chacun d'entre eux s'appliquant un territoire particulier. Si une telle perspective a un sens pour les formations généralistes, chaque établissement de formation (des lycées professionnels aux STS - sections de technicien supérieur - ou aux universités) qui offre des enseignements spécifiques a sa propre aire de recrutement, non disjointe des autres et le plus souvent les chevauchant. Mais la mobilité géographique à la sortie de l'appareil de formation pour l'insertion professionnelle a permis de montrer la prégnance des modèles classiques de diffusion, c'est-à-dire que plus l'on s'éloigne des bassins de vie initiaux (école, domicile) plus la densité des emplois trouvés diminue: là, comme souvent, la distance école-emploi est un facteur explicatif important de l'insertion professionnelle. 
Souvent un arbitrage est réalisé entre maintien dans la spécialité du diplôme et distance au lieu de socialisation initiale. Il y a bien un territoire de l'insertion. Les zones d'emploi en fournissent parfois une approximation pertinente suffisante, en particulier pour les qualifications les moins élevées.

Se dégagent ainsi d'autres compréhensions du « territoire ». Celui-ci n'est plus le simple décalque du découpage administratif, mais il résulte des comportements migratoires des individus. Mais cette nouvelle conception d'un espace géographique pertinent associe une recherche statistique des mobilités avec un secteur particulier de la politique publique (l'emploi, la formation). La difficulté est que ces découpages fonctionnels ne sont pas toujours transmissibles à d'autres domaines de l'action publique. Par exemple, le découpage en zones d'emploi n'a aucune pertinence pour le domaine de l'agriculture, plus sensible à des unités agro-paysagères. Chaque acteur peut ainsi faire valoir sa propre logique de structuration de l'espace et l'on ne doit pas s'étonner de leur multiplication. Ces découpages, pour pertinente que soit leur logique, ne permettent pas toujours la coordination des acteurs et la formation de connaissances partagées.

Néanmoins, les zones d'emploi ont acquis une importante visibilité qui a pu leur conférer une légitimité pour fournir une définition de l'unité de base du territoire. Il n'en reste pas moins que cette légitimité a pu être contestée par l'effet même du développement économique. Le « territoire » des entreprises n'est pas celui de l'action publique.

\section{TERRITOIRES ET ENTREPRISES : DU LOCAL ET DE LA PROXIMITÉ}

Les zones d'emploi permettent une description de l'espace à partir des seules mobilités des salariés. C'est évidemment une très grande simplification. S'intéresser aux entreprises permet de penser l'espace de manière différente : leur organisation, la répartition du pouvoir en leur sein pèsent sur leur localisation et celle de leurs établissements. On retrouve les deux dimensions verticale et horizontale, non plus comme expression des politiques publiques et des découpages administratifs, mais en relation à l'organisation des entreprises et à leur insertion dans les territoires.

\section{Des transformations organisationnelles majeures}

Les transformations organisationnelles des entreprises ont été importantes depuis une trentaine d'années. Sans vouloir être exhaustif, on rappellera certains faits. Ils témoignent tous d'une remise en cause du modèle fordien.

Tout d'abord, les grandes concentrations ouvrières ont assez sensiblement diminué au profit d'établissements de plus petite taille. D'une part, le recul de l'emploi industriel s'est manifesté, notamment pour les emplois peu qualifiés. Mais aussi, pour des raisons de gestion, les établissements qui se maintiennent ont des tailles plus réduites : ils sont plus nombreux mais moins dispersés en zone rurale et plus concentrés en zone urbaine et périurbaine.

Le modèle fordien d'organisation des entreprises s'est développé en privilégiant l'organisation hiérarchique d'entreprises fermées sur leur environnement, cherchant à minimiser leur dépendance au territoire. Le pouvoir concentré dans des groupes financiers ou aux sièges sociaux et des établissements périphériques dominés, la gestion des entreprises privilégiait les ressources internes et une planification rigide. Ces hiérarchies organisationnelles et la répartition fonctionnelle des entreprises et des établissements sur l'ensemble du territoire relevaient d'une logique verticale sans rapport avec la hiérarchie administrative, et autonome par rapport à elle : produit de l'histoire et des multiples fusions et cessions, la localisation des entreprises ressortait pour partie de l'arbitraire, laissant souvent insaisissable le lieu réel de leur pouvoir décisionnel. Ce modèle s'est considérablement modifié.

Les entreprises ont vu leurs fonctions se complexifier : le simple comptable a dû se transformer en gestionnaire, le chef du personnel en directeur des ressources humaines. Cette complexification s'est accompagnée d'une externalisation de ces fonctions. Les entreprises font désormais appel à des entreprises spécialisées : cabinets d'expertise comptable ou de 
recrutement, publicitaires, juristes, ingénieurs conseil, centres de recherche et de transfert, pour des services de haut niveau. Mais elles sollicitent également des entreprises de nettoyage, de location de véhicules, de gardiennage ou de restauration. L'ensemble de ces services est désormais indispensable aux entreprises : elles deviennent plus dépendantes de la localisation et de l'accessibilité de ces services. Il en est de même dans le domaine de l'emploi et de la formation. Entre la main-d'œuvre et l'entreprise, il existe désormais un grand nombre d'intermédiaires, privés (sociétés d'intérim ou de formation) ou publics (ANPE - Agence nationale pour l'emploi -, ...). Ces intermédiaires ont des localisations souvent urbaines : les agglomérations ou les aires urbaines apparaissent alors comme les territoires pertinents pour la prise en compte de ces phénomènes d'externalisation.

Selon le niveau des fonctions en cause, ces services spécialisés ont une localisation contrainte : elle peut aller de la plus stricte proximité à l'entreprise à une concentration de type métropolitain dans les plus grandes agglomérations. Quoi qu'il en soit, la localisation de ces services est aujourd'hui un facteur déterminant du développement territorial, et leur densité autour de l'entreprise un élément de leur choix de localisation. À ce titre, les organismes publics comme les entreprises privées (souvent de forme associative) de formation jouent un rôle important.

\section{Des spécialisations territoriales d'entreprises}

Le recul du mode de production fordien a entrâné le développement de la production flexible. Elle s'est imposée soit en raison de la nature même des produits soit comme conséquence des changements technologiques et de l'informatisation de la production. Des modifications importantes dans la gestion de la main-d'œuvre ont accompagné ces transformations productives : d'une manière générale, elles ont induit, d'une part, une hausse des qualifications requises et, d'autre part, une autonomie décisionnelle et une implication plus grandes de la part des salariés. L'offre de formation, souvent locale, initiale et continue, est ainsi devenue une des variables conséquentes dans la gestion de la main-d'œuvre et son adaptation aux nouvelles exigences concurrentielles.
Ces transformations vers une production plus flexible ont pris des formes multiples, mais toutes ont conduit à réévaluer le rôle du territoire dans le développement. Plus exactement, territorialisation et mondialisation sont devenues les deux pôles complémentaires du développement des entreprises. On a pu définir ces deux pôles à travers l'opposition local/global. Souvent, la nouvelle dynamique territoriale s'est exprimée par la forme prise par la contrainte « locale»: les «économies de la proximité » ont fait parfois l'impasse sur les conséquences " géographiques » liées à la mondialisation et ses réseaux métropolitains. Celles-ci ont imposé des critères de localisation fondés sur des potentialités d'accessibilité, présentes dans les environnements métropolitains. Ainsi, c'est avec une certaine confusion que les « économistes de la proximité » ont pu définir le «local» par la référence à des proximités géographiques (horizontales), mesurées donc par des distances kilométriques et distinguées des proximités (verticales) organisationnelles ou sociales. Il y a eu confusion préjudiciable entre les termes « géographie » et « local », la géographie ne se limitant pas aux relations de proximité. Elle s'intéresse tout autant au « global » qu'au « local ».

Au-delà de ces ambiguïtés sémantiques, l'observation de spécialisations territoriales flexibles et de leur efficacité productive a été le vecteur de la réintroduction du territoire dans l'analyse économique. Les districts industriels ont montré, en effet, des formes productives spécifiques associant sur un même territoire une multiplicité d'entreprises de même activité (habillement, chaussures, meubles,...). Ce que l'on a appelé la «Troisième Italie», située entre Rome et Milan, a été la figure emblématique de ce modèle productif. Le choix de ce mode de production flexible et dynamique, caractérisant des espaces géographiques homogènes, aux relations sociales structurées et spécifiques, a été replacé dans une perspective économique marshallienne qui n'est pas sans évoquer aussi la vieille notion de «milieu » de Vidal de la Blache (cf. Ozouf-Marignier, 1992). Décolletage de la Vallée de l'Arve, plasturgie à Oyonnax, chaussure à Cholet, lunetterie à Morez, etc., ont été les figures emblématiques de ce retour sur l'histoire économique à côté d'une évocation des spécialités agro-alimentaires localisées. 
Des formes multiples de ces territoires spécialisés ont été identifiées, sinon mythifiées : districts industriels, milieux innovateurs, technopôles ont ainsi été convoqués, chacun à leur tour, comme gages de la modernité. Les politiques territoriales d'État (la Délégation à l'aménagement du territoire : DATAR, devenue la Délégation interministérielle à l'aménagement et à la compétitivité des territoires : DIACT) s'en sont emparées et ont assuré la promotion des Systèmes productifs locaux (SPL) ou des Pôles de compétitivité. Ces deux outils de l'aménagement du territoire, pour différents qu'ils soient, mettent ensemble l'accent sur les nécessaires relations territoriales (horizontales) des entreprises entre elles et avec les institutions publiques, dans l'idée d'encourager des processus d'innovation. C'est donc aujourd'hui autour de la production scientifique et de son transfert dans les processus productifs que la question du «territoire » est posée. Elle affecte indirectement l'offre de formation, elle aussi fortement localisée. Des segments spécifiques de cette offre sont plus fortement concernés : écoles d'ingénieurs, formations professionnelles universitaires, STS apparaissent comme des interfaces possibles entre laboratoires de recherche, offre de formation et entreprises innovantes. Par effet de contagion et de diffusion, l'ensemble du dispositif de formation professionnelle est entraîné dans cette logique où une offre localisée, adaptée aux besoins des entreprises de ces systèmes productifs locaux, doit répondre aux exigences de modernisation de l'appareil productif: celui-ci est entré dans l'horizon du «capitalisme cognitif». La formation professionnelle comme la recherche doivent être mobilisées pour la nécessaire modernisation des entreprises. Les spécialisations territoriales dans la recherche et l'innovation sont une des conditions de la réussite de cet objectif : la tentative est faite de promouvoir une adéquation plus forte de la recherche et de la formation aux besoins des entreprises locales.

\section{Pourquoi le «local » et la proximité ?}

De nombreuses analyses ont conduit à cerner les arguments théoriques justifiant de l'efficacité de ces systèmes localisés : le développement de coopérations horizontales entre les entreprises elles-mêmes et entre les entreprises et des structures publiques en serait la garantie. Deux types d'arguments peuvent être évoqués succinctement, parmi d'autres.

D'une part, la proximité permet de bénéficier des externalités fournies par les équipements publics localisés : ces biens collectifs permettent des gains de productivité à ceux qui peuvent en bénéficier. Ces biens peuvent aussi être des "actifs spécifiques» détenus par les acteurs locaux (patrimoine naturel, savoir-faire, accessibilité,...) Les coûts de transport et plus généralement les coûts de transactions diminuent avec la proximité, qui permet de maximiser les gains d'externalités.

Ces mêmes coûts de transactions peuvent être mobilisés pour justifier les limites à l'intégration et expliquer la persistance d'une multiplicité d'entreprises indépendantes; dès lors, les relations suivies entre ces entreprises, entre concurrence et coopération, s'éloignent des purs mécanismes concurrentiels : des coopérations non marchandes peuvent apparaître, pour autant que des relations de confiance, socialement structurées, se mettent en place et que des habitudes de travail et des routines organisationnelles révèlent l'efficacité des contrats incomplets. Les jeux coopératifs des acteurs renforcent les dynamiques territorialisées.

D'autre part, la circulation de l'information et la production de l'innovation gagneraient à la proximité. Cette information peut être décrite sous deux modalités : la première, formelle ou digitale, peut être codifiée et transférée sous forme universelle (un théorème mathématique) et/ou écrite (livres, lettres, fax, Internet). La seconde, tacite et informelle, plus analogique, s'exprime plus efficacement dans les relations orales et pragmatiques des individus entre eux. Elle suppose le face-à-face réel entre individus se rencontrant véritablement, c'est-à-dire dans un même lieu.

Cette dernière modalité, nécessitant les rencontres, la connaissance des partenaires entre eux, des relations amicales répétées, s'exprime plus facilement grâce à la proximité (il y a bien sûr des contre-exemples, la proximité attisant et figeant parfois les conflits).

De multiples arguments complémentaires peuvent être évoqués qui expliquent les concentrations et spécialisations territoriales comme du reste les 
ségrégations sociales qui semblent bien se renforcer, en raison même du développement des formes de libéralisation et de déréglementation des échanges.

Les justifications de cette proximité mériteraient d'autres explications, en particulier pour différencier les facteurs de spécialisation territoriale de ceux proprement de métropolisation. Il n'en reste pas moins que les territoires se développent en se différenciant dans leurs structures comme dans leurs dynamiques. C'est ainsi que la «métaphore de l'archipel » a pu être évoquée pour décrire les économies contemporaines. Mais il faut aussi se souvenir qu'aucune île ne ressemble vraiment à une autre ni par sa taille ni par sa géographie. Et que si « des régions gagnent », c'est logiquement parce que d'autres perdent.

\section{CONSTRUIRE PAR LA FORMATION DES TERRITOIRES COMPÉTITIFS}

La reconnaissance du fait «territoire » s'accompagne des effets de la croissance inégale des régions ou des espaces. Ces inégalités proviennent sans doute du patrimoine historique légué (naturel ou culturel), mais surtout des jeux plus ou moins coopératifs des acteurs et de leur capacité à mobiliser les ressources locales dans une perspective innovante, ou du rôle d'un ou de plusieurs leaders ou porteurs de projets.

Dans une perspective orthodoxe, on peut dire que l'émergence d'un territoire procède d'un mécanisme de différenciation particulier mais classique : il s'exprime sur un espace donné, il implique une possibilité de différenciation spatiale. Son effet ultime est de créer un mécanisme de protection par des barrières non tarifaires (des externalités, équipement ou circulation des informations); en d'autres termes, les mécanismes de concurrence imparfaite, générés par les ressources territoriales et leur mobilisation, permettent une efficacité productive accrue, rémunérant mieux les facteurs de production locaux et incitant les agents économiques à investir sur ce territoire.

Linefficacité de ces mécanismes se lit inversement dans les phénomènes de délocalisation. Si des entre- prises quittent le territoire, c'est que les mécanismes de protection et d'innovation sont insuffisants. Ces délocalisations jouent aujourd'hui à deux niveaux. D'une part, les activités peu qualifiées, standardisées ou génériques, sont confrontées à des concurrences par le coût du travail. Les fermetures d'entreprises ont d'abord touché les emplois des entreprises industrielles fordiennes, mais atteignent aujourd'hui des qualifications moyennes et des emplois tertiaires. D'autre part, des concentrations d'activité s'observent autour des pôles de recherche et développement et des industries de haute technologie qui maintiennent leur développement.

Entre ces deux niveaux, les perspectives sont plus incertaines, mais pour qu'elles soient favorables, une augmentation des qualifications et un développement des processus d'innovation sont nécessaires. La base territorialisée de ces évolutions est désormais recherchée. Produire du «territoire », c'est s'assurer contre les risques de délocalisation. Les liens entre formation et territoire sont donc décisifs.

D'une part, l'offre de formation est très fortement localisée et cette localisation dépend autant du découpage administratif que des densités de population. Le rôle de l'École et de la formation professionnelle (publiques) est d'offrir à ses usagers (les élèves et les étudiants) les formations pour lesquelles ils sont motivés et qu'ils désirent suivre. Du reste, une partie des formations est générique et formelle (par exemple, les formations de comptabilité n'ont en elles-mêmes rien de local). Dans cette perspective, le système de formation doit aussi assurer la mobilité des élèves et leur insertion professionnelle ne saurait être uniquement locale.

Mais par ailleurs, il doit tisser des liens et développer des relations avec le milieu économique local: formations d'initiative locale, apprentissage, stages en entreprises, etc.

D'autre part, le développement des territoires passe le plus souvent (en l'absence d'une activité ou d'une entreprise encore dominante) par les coopérations entre les entreprises locales et par leurs capacités à développer des actions collectives. Que ces relations restent encore dominées par des relations de marché au sein des larges espaces métropolitains ou que des formes de coopérations plus étroites puissent se 
développer sur des territoires plus restreints, leurs densités est une des conditions de la réussite. Mais ces entreprises sont confrontées au double problème de l'innovation et de la formation de la maind'œuvre: leur capacité à trouver des ressources qualifiées est en question. Cela ne peut se réaliser que par des coopérations avec les structures de formations initiale et continue, encore largement liées à la puissance publique. En particulier, les nécessaires anticipations des métiers du futur sont souvent trop mal documentées par les entreprises et leurs organisations professionnelles. C'est pourtant une des clés de l'adaptation territorialisée de l'offre de formation.
C'est dans ce champ d'anticipation des besoins futurs que s'expriment le mieux les nécessités d'une planification locale de l'offre de formation. Que cette compétence ait été confiée aux conseils régionaux, qu'ils jouent pour cela le rôle de pivot entre les différents acteurs, incite aussi à valider l'idée que la territorialisation de l'offre de formation est incontournable, sans que le territoire soit conçu de manière trop stricte comme un zonage. Le «territoire» est le produit d'actions multiples, d'efforts et de coopérations entre public et privé, parmi lesquels les enjeux de la formation jouent un rôle crucial. Finalement, comme le développement économique, le « territoire » se mérite.

\section{Bibliographie}

Benko G., Lipietz A. (1992), Les régions qui gagnent, PUF.

Benko G., Lipietz A. (2000), La richesse de régions, PUF.

Camagni R., Maillat D. (2006), Milieux innovateurs, Économica.

Caro P., Hillau B. (1997), « La logique dominante des publics scolaires : offre de formation et environnement local », Formation Emploi n ${ }^{\circ}$ 59, La Documentation française, pp. 87-103.

Formation Emploi (2004), Dossier « Ces territoires qui façonnent l'insertion », $\mathrm{n}^{\circ} 87$, juillet-septembre.

Ozout-Marignier M. (1992), «Géographie et Histoire ", in Bailly A., Ferras R., Pumain D., Encyclopédie de la géographie, Économica.
Pecqueur B. (Éd.) (1996), Dynamiques territoriales et mutations économiques, L'Harmattan.

Pecqueur B., Zimmerman J.-B. (2004), Économie de la proximité, Hermès.

Pumain D., Saint-Julien Th., (2001), Les interactions spatiales, Cursus Armand Colin.

Timotéo J., Vernières M. (Éds.) (2002), Dynamiques du local. 10 ans de recherche sur l'approche localisée de la relation formation emploi, Journées d'études centres associés au Céreq-MATISSE, 25 et 26 octobre 2001, Paris, Céreq, collection « documents $\mathrm{n}^{\circ}$ 167, série Séminaire », $237 \mathrm{p}$. 